Candice Que - Ansorge, MD

Eduardo C. Yap, MD

Metropolitan Medical Center

Department of Otorhinolaryngology

Head and Neck Surgery

\section{The Use of Bony Septum as an Extended Spreader Graft in Primary and Secondary Rhinoplasty}

\begin{abstract}
Objective: To describe a surgical technique using bony septum specifically vomer or perpendicular plate of the ethmoid (PPE) as an extended spreader graft (ESG) for securing septal extension graft (SEG) and for correcting internal nasal valve dysfunction.
\end{abstract}

Methods:

\section{Design: Descriptive Case Series \\ Setting: $\quad$ Tertiary Private Hospital}

Participants: Thirty-two (32) patients who underwent aesthetic rhinoplasty from May 2016 to October 2017 were evaluated and ten (10) patients presenting with symptomatic obstruction were considered for inclusion. The surgical technique was applied in patients with weak SEG for control of nasal length and tip projection who had inadequate septal cartilage for SEG and ESG intraoperatively. Results were evaluated grossly under direct vision intra-operatively and post-operatively to check the patency of the internal valve.

Metropolitan Medical Center

Department of Otorhinolaryngology

Head and Neck Surgery

1357 G. Masangkay St, Santa Cruz, Manila 1012

Philippines

Phone: 0947-7474007

Email: candiceque@yahoo.com

The authors declared that this represents original material that is not being considered for publication or has not been published or accepted for publication elsewhere in full or in part in print or electronic media; that the manuscript has been read and approved by the authors, that the requirements for authorship have been met by each author, and that the authors believes that the manuscript represents honest work.

Disclosures: The authors signed a disclosure that there are no financial or other (including personal) relationships, intellectual, passion, political or religious beliefs, and institutional affiliations that might lead to a conflict of interest.

Presented at the Cesar F. Villafuerte Sr. Research Contest (3rd Place). November 30, 2017, Maynila Ballroom, Manila Hotel.

Presented at the Antonio L. Roxas International Research Contest (3rd Place). December 1, 2017, Maynila Ballroom, Manila Hotel.

Presented at the 10th International Academic Conference in Otology, Rhinology and Laryngology. March 2, 2018, Fairmont Hotel. 
The septal extension graft is frequently used to lengthen the tip of the nose among Filipinos. The relatively larger portion of harvested cartilage is allotted as septal extension graft. To compensate for lack of support, a small reinforcing batten graft is usually added on the contralateral side, allowing further stabilization of the septal extension graft and preventing the gradual deviation of the graft. ${ }^{1}$ It is commonly encountered in Filipinos that the fixation strength seems insufficient and additional extension is needed. More often than not, internal valve dysfunction is also encountered in primary and secondary rhinoplasty. In both instances, an extended spreader graft may be used. Our literature search showed the use of bony septum as a batten graft ${ }^{2,3}$ or as a septal extension graft ${ }^{4}$ but to the best of our knowledge, no study has yet described the use of bony septum as an extended spreader graft.

This study aimed to describe a surgical technique using bony septum, specifically vomer and perpendicular plate of the ethmoid, as an extended spreader graft (ESG) to secure the septal extension graft (SEG) in selected patients that needed overcorrection, to control the nasal length and to avoid post-operative nasal shortness, to avoid tip deviation or rotation, as well as to improve the breathing of the patient.

\section{METHODS}

This is a descriptive case series of a surgical technique using the bony septum (specifically vomer and perpendicular plate of the ethmoid) as an extended spreader graft in a tertiary private hospital with IRB approval from the Manila Central University Filemon D. Tanchoco Sr. Medical Foundation Institutional Review Board (MCUFDTMF IRB). A total of thirty-two (32) private patients of the authors seeking aesthetic rhinoplasty (primary and secondary cases) that were operated from May 2016 to October 2017 were screened. With informed consent, 10 patients with nasal obstruction were evaluated by the authors for inclusion. Inclusion criteria for this technique were (1) presence of symptomatic nasal obstruction on initial consultation and (2) inadequate septal cartilage harvested for SEG and ESG usage. Exclusion criteria were (1) patients with no symptoms of obstruction, and (2) symptomatic patients who had adequate septal cartilage harvested for SEG and ESG intraoperatively. The nasal obstruction was pre-operatively evaluated under direct vision via anterior rhinoscopy and by Cottle maneuver.

All rhinoplasties were performed via an open approach. Conchal cartilage was harvested for tip reconstruction. As previously described, a modified alar lift procedure via "sail" excision was done in patients with hanging ala, which is commonly seen in Southeast Asian noses..$^{5}$ The alar rim was lifted by removing a triangular piece of tissue in the inner lateral vestibular skin. ${ }^{5.6}$ Dissection of the lower lateral cartilage was approached at the medial crura, then at the lower lateral cartilage beneath the superficial muscular aponeurotic system (SMAS) and finally at the dome. ${ }^{5}$ The skin and soft tissue envelope of the septum was dissected in the subperichondrial plane anteriorly until subperiosteal elevation of the nasal bone. The dorsal septum was dissected along the submucoperichondrium layer until the bony-cartilaginous junction was reached. Membranous septum was dissected to access the caudal edge of the septum. Harvesting of the nasal septum via an anterior septal approach caudally was done using a Freer septum D-knife $5 \mathrm{~mm} \times$ x 8mm blade (Storz N2252, Karl Storz, Germany) or a Freer septum elevator, leaving sufficient septum dorsally and caudally for the L-shaped strut. The bony septum, which was either vomer or perpendicular plate of the ethmoid, was cut using septal scissors along the dorsal septal strut and maxillary crest (Figure 1) and fractured using Freer elevator or Takahashi forceps. If septal scissors were not available, Mayo scissors could be used. Using $1 \mathrm{~mm}$ ophthalmic burr tip, $0.5 \mathrm{~mm}$ holes were drilled on both sides, anteriorly and posteriorly, about $2 \mathrm{~mm}$ from the edge for anchoring. (Figure 2) If a burr tip was not available, a G20 peripheral intravenous needle could be used instead to produce holes. The septal extension graft was anchored on one side of the caudal septum with three or four sutures using polydioxanone PDS $\| 5.0$ with $13 \mathrm{~mm}$ reverse cutting needle (Ethicon, Somerville, NJ, USA) for fixation while the vomer or perpendicular plate of the ethmoid was fixed on the contralateral side. (Figure 3 and 4) Tip grafts (such as shield, onlay and backstop) were placed according to the needs of the patient. Osteotomy was done in patients with an inverted- $\mathrm{V}$ deformity or broad bony vault. Dorsal implants were designed using a sheet of preformed expanded Poly Tetra Fluoro Ethylene ePTFE, inserted and fixed such that no depression or irregularities were noted. Closure of incisions were done meticulously. As a last step, alarplasty was performed when needed.

The results were evaluated grossly under direct vision intraoperatively and immediately post-operatively to check the patency of internal valve. The patients were asked at 2 and 4 weeks postoperatively to rate the results as worse (more obstructed), same, or improved (better breathing). Pre- and post-operative photographs at 2 weeks were evaluated for improvement in lengthening of nose and projection of nasal tip. All patients were jointly and simultaneously evaluated by both co-authors.

\section{RESULTS}

Of the thirty-two (32) patients who underwent rhinoplasty from May 2016 to October 2017, twenty-two (22) presented with no symptoms of obstruction and were excluded from this series. Ten (10) presented with 


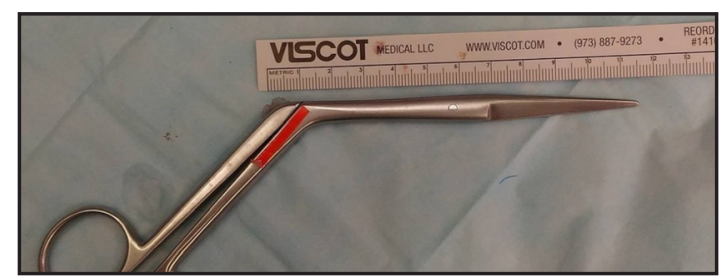

Figure 1. Septal scissors used

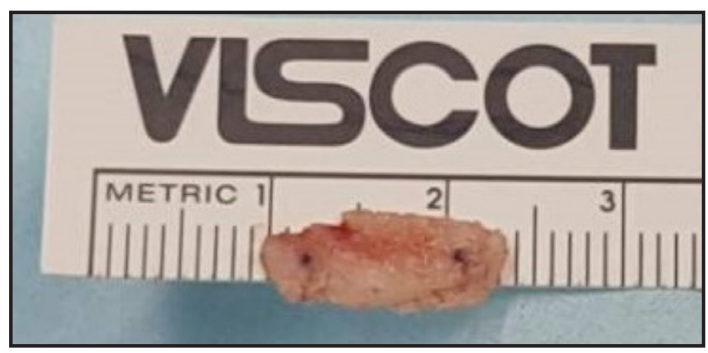

Figure 2. Vomer with burred holes

symptomatic obstruction and were considered for inclusion. Among the symptomatic patients, five (5) had adequate septal cartilage for SEG and ESG and were further excluded, while bony septum was harvested and used as an ESG in five (5) patients with inadequate septal cartilage who were finally included in this series. These five (5) included 1 male and 4 females whose ages ranged from 35 to 50 -years-old (mean age $41.8 \pm 5.3$ years).

Intraoperative evaluation under direct vision showed the anterior caudal septum was deviated in all 5 patients at the area of the internal valve, and correction was confirmed after placement of SEG and ESG.

The use of bony septum (specifically vomer and perpendicular plate of the ethmoid) as an extended spreader graft was able to secure and strengthen the septal extension graft in patients that needed overcorrection of nasal tip. The tip was adequately lengthened and derotated, which prevented post-operative nasal shortness.

Immediate post-operative evaluation further confirmed the nasal valve was bilaterally patent in all 5 patients and all 5 patients reported subjectively improved breathing or relief of obstructive symptoms when asked to rate the breathing as worse, same, or improved, 2 and 4 weeks post-operatively. Post-operative photographs showed improvement of nasal length and tip. (Figure 5)

\section{DISCUSSION}

Our small series demonstrated that the bony septum (vomer and perpendicular plate of the ethmoid) was able to act as an extended spreader graft to stabilize the septal extension graft, correcting anterior septal deviation and nasal valve obstruction. It improved breathing, nasal length. Since the new nasal tip is secure, this should prevent long term tip rotation.

In rhinoplasty, facial aesthetic analysis is critical for proper surgical
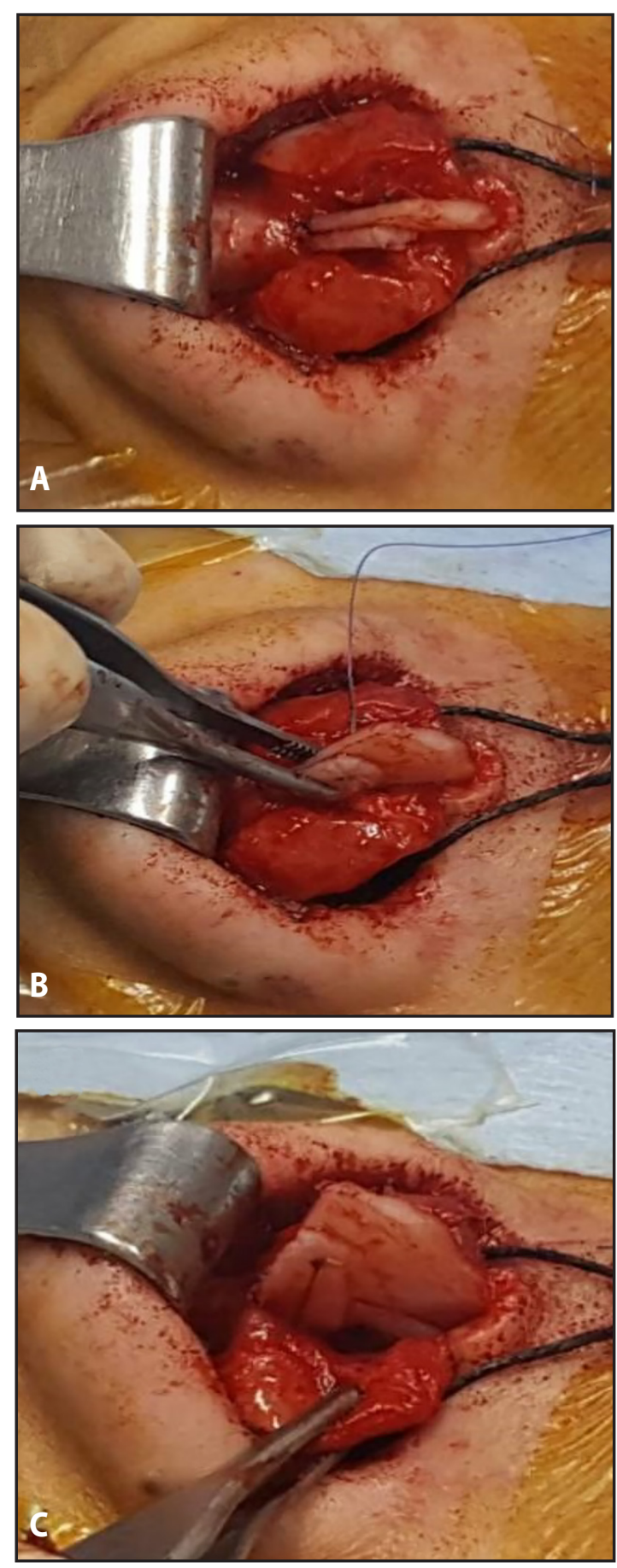

Figure 3. A. Placement of SEG B. Fixation of SEG and C. Placement and securing of vomer as Extended Spreader Graft (ESG)

planning. Parameters such as rotation and nasal projection should be carefully evaluated. The amount that the tip of the nose is turned up or down is referred to as "rotation" and is evaluated with the nasolabial angle. In females, the angle must be obtuse, from 95-105 degrees, whereas for a male, the ideal is nearly forming a right angle, from 90- 


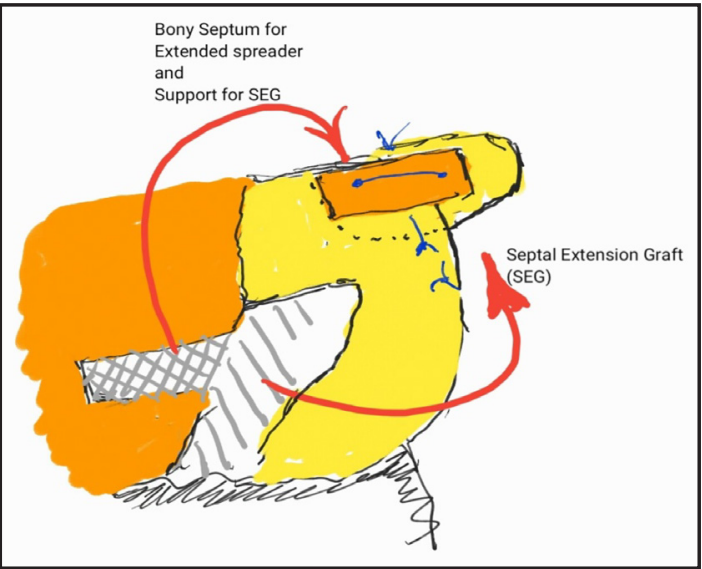

Figure 4. Illustration showing use of bony septum as ESG and cartilaginous septum as SEG

95 degrees. $^{7}$ It becomes unattractive when too much of the nostril is visible from the frontal view. In rhinoplasty, rotation means turning the tip of the nose up while counter-rotation means turning the tip down. Counter-rotation is more commonly done in Filipino noses and presents a much greater surgical challenge. It has the effect of lengthening a shortened nose. Cartilage is used to fashion grafts for this purpose. The commonly used grafts for tip repositioning and recontouring of the dorsal nasal vault are septal extension graft, caudal septal extension graft, spreader graft, extended spreader graft and a variety of onlay grafts.

Septal extension graft (SEG) is used as a standard method for Asian nasal tip surgery for tip projection, tip rotation or counterrotation, and better tip shape. Kim et al' cited that the graft is a suitable method to extend the Asian nose and to maintain the thick skin since it provides both a direct extension of the framework and maintains a strong support. Byrd et al. ${ }^{8}$ were the first to introduce 3 types of septal extension grafts (paired spreader grafts, paired batten grafts and direct extension grafts), depending on the nasal tip position. These overlapping grafts provide more support and prevent deviation. Disadvantages are having a stiff nasal tip and a thick membranous septum postoperatively. This graft is the workhorse used in Filipino noses.

A modification popularized by Toriumi et al. was the use of a caudal extension graft that is longer along the superior margin to push the nasal tip down for counter-rotation. ${ }^{9}$ This is also commonly used in Filipino noses since it makes the graft longer along the inferior margin, increasing tip rotation and blunting the nasolabial angle, which are common problems with Filipino noses.

In 1984, Sheen first introduced the use of spreader graft, widely used for functional and cosmetic purposes. ${ }^{10}$ Since a lot of Filipinos lack
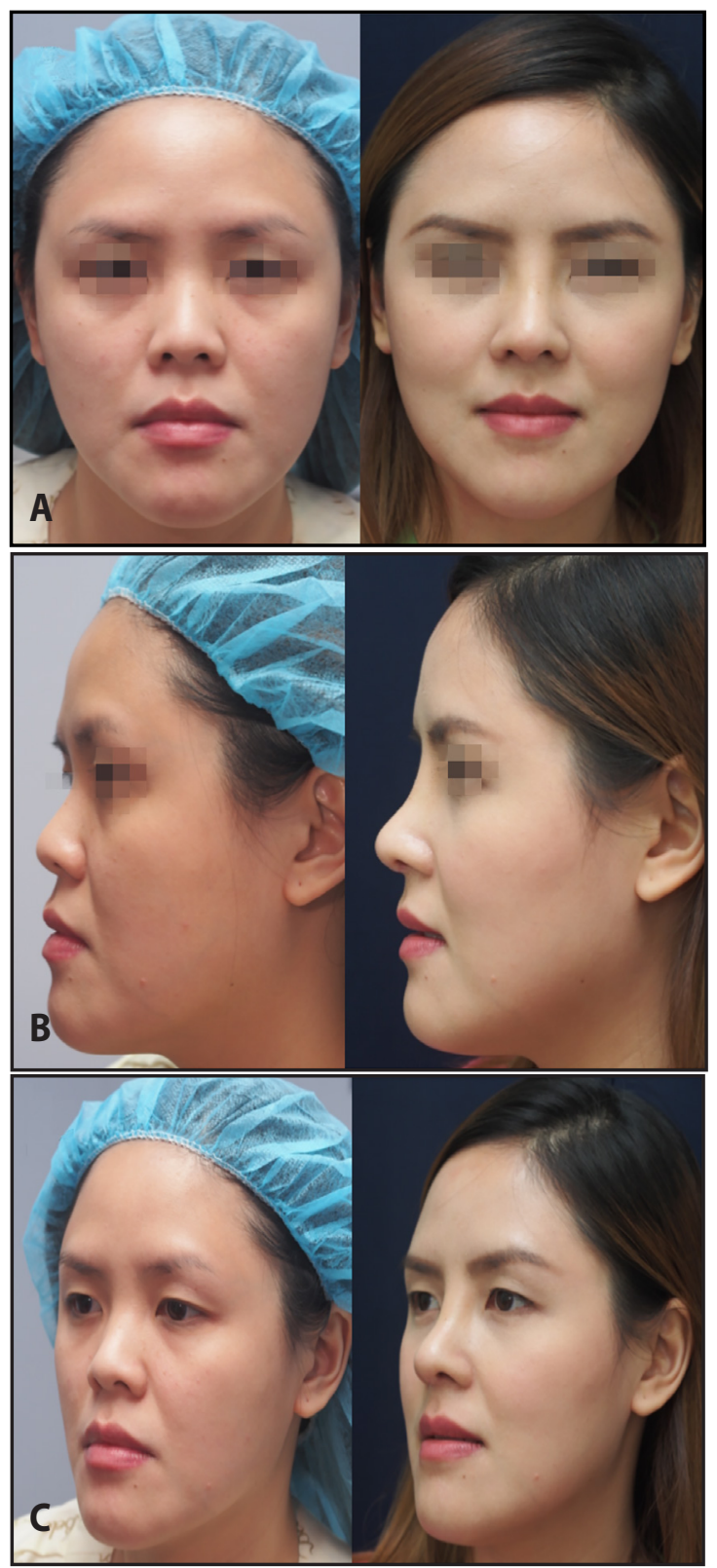

Figure 5. A 36-year-old woman with oriental nose who underwent primary rhinoplasty, pre- and post-operative photos: A. Frontal B. Lateral; and C. Left oblique views (photos published with permission).

dorsal support, its advantage is to correct the lack of dorsal support to lateral walls in internal nasal valve dysfunction and to restore a normal dorsal profile in narrowed or collapsed nasal vault. ${ }^{10}$

Extended spreader grafts are frequently used to enhance nasal tip support. Acting as a splint graft, they may be placed on each side of SEG to stabilize it and to ensure that the caudal septum is straight. These grafts are placed between the dorsal septum and the medial edge of the upper lateral cartilage and fashioned to extend into the tip-lobule complex to help alter tip position and definition. This ensures 


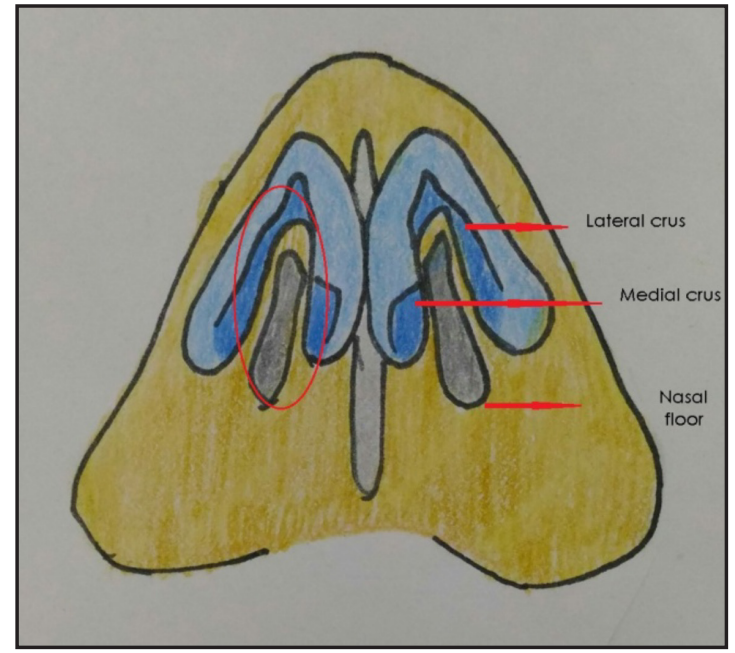

Figure 6. External valve illustration where nasal obstruction might occur

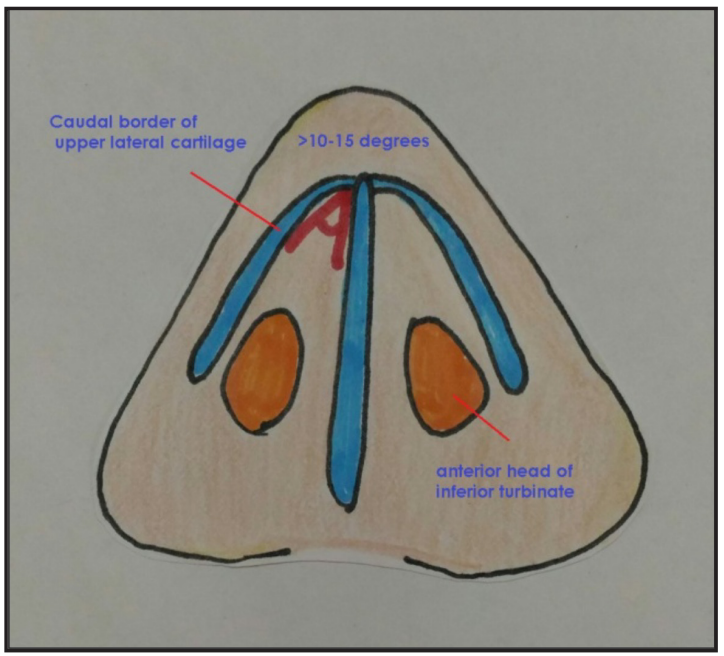

Figure 7. Internal valve illustration where nasal valve collapse might occur

the dorsal septum is strengthened and straightened, internal valve is widened and tip projection is improved. ${ }^{11}$ It also aids in preserving nasal length subsequently avoiding the development of a short nose deformity post-operatively. ${ }^{12}$

In 1903, Mink first described the nasal valve, dividing it into external and internal portions. It is important to know its anatomy to recognize if the nasal obstruction is from the external valve or internal valve. The external nasal valve (Figure 6) is formed by the columella, the nasal floor and the nasal rim or caudal border of the lower lateral cartilage. ${ }^{13}$ The internal nasal valve area superolaterally is the caudal border of upper lateral cartilage, medially the septum, inferiorly the floor of pyriform aperture and posteriorly the head of inferior turbinate. The internal nasal valve (Figure 7) is the specific structure within the internal nasal valve area between the caudal border of upper lateral cartilage and

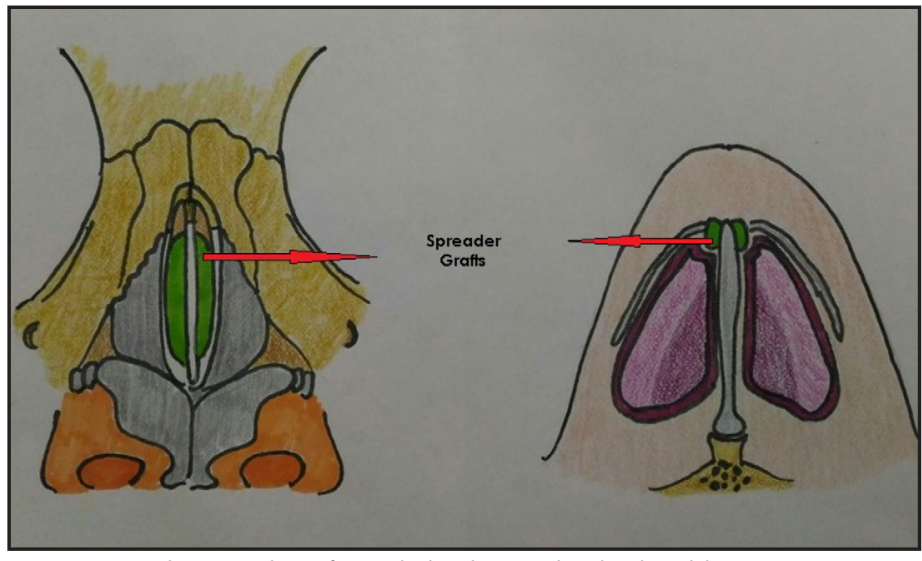

Figure 8. Area where spreader graft is applied as shown in dorsal and caudal view

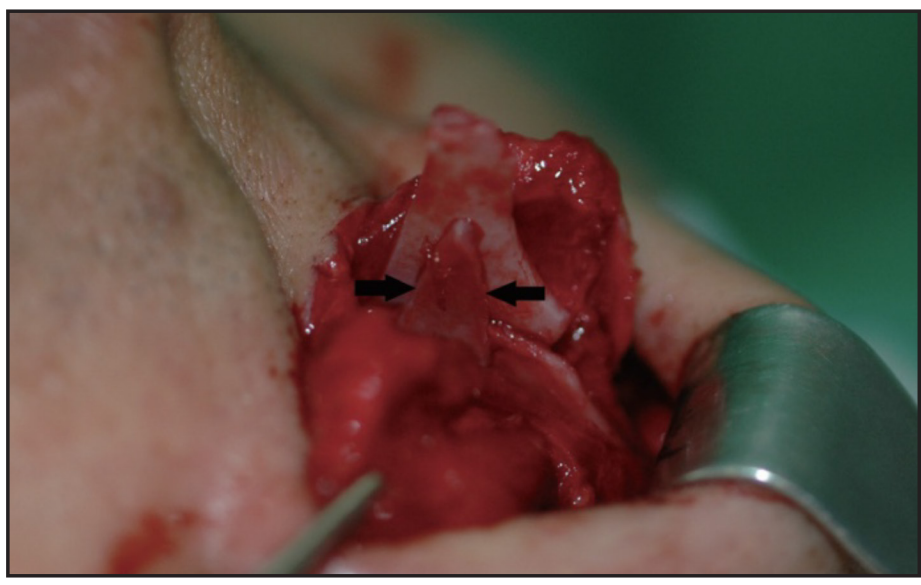

Figure 9. Vomer used as batten graft to correct caudal septal extension

septum. It is 10 to 15 degrees in Caucasian nose and wider in African or Asian nose. If the angle is less than 10 degrees, nasal obstruction may ensue. ${ }^{11}$ A CT scan study by Suh et al. ${ }^{14}$ confirmed the internal nasal valve angle in Asians to be significantly larger than in Caucasians with a measurement of 21.6 degrees $+/-4.5$ degrees.

Relief of nasal obstruction is another concern in this study. In patients with internal nasal valve collapse, unilateral or bilateral nasal obstruction is the primary complaint. Some present without nasal obstruction and just evidence of aesthetic asymmetry. Other factors to be considered are septal deviation, inferior turbinate hypertrophy and external nasal valve collapse. According to Helinski, ${ }^{11}$ examination may reveal an asymmetric brow tip aesthetic line or pinched middle vault seen on frontal view. He also mentioned that in cases of prior reductive rhinoplasty, patients may present with an inverted $V$ deformity. In developed countries, acoustic rhinometry is performed and is considered the objective standard to detect obstructions in specific sites of the nasal cavity. Sound waves are used to determine whether the valve area in the nasal cavity is too narrow. In less developed 
countries, diagnosis is with visualization of the valvular area, ideally with the use of a zero-degree nasal endoscope. It can also be visualized with anterior rhinoscopy or the use of a nasal speculum as was performed in the patients. Application of nasal decongestant can be performed to aid in identifying reversible mucosal edema. However, Helinski stated that examining the valve without disturbing it with a nasal speculum is important because the speculum usually distorts the relationship of the septum and the caudal edge of the upper lateral cartilage and opens the valve artificially. ${ }^{11}$ A practical method that would not alter this anatomical relationship is to elevate the tip of the nose gently with the examiner's finger with inspection using a good light source. ${ }^{11}$

A quick and easy test, the Cottle maneuver is used to evaluate internal nasal valve stenosis or disorder. ${ }^{15}$ The cheek of the evaluated side is gently pulled laterally with 1-2 fingers and the patient is asked to breathe. A positive test result is when the obstruction is relieved. A more specific and reliable test is the modified Cottle maneuver wherein a thin instrument such as cotton swab or cerumen curette is placed at the level of the external nasal valve and internal nasal valve. This is an accurate method to identify the level of obstruction

The purpose of the spreader graft is to open the internal valve and to correct the septal deviation. (Figure 8) An extended spreader graft on the other hand is used as support or reinforcement for the septal extension graft and for securing it, as well as to address internal nasal valve dysfunction.

In this study, patients in a tertiary private hospital needing additional support of the L-strut and presenting with internal valve problems were included. This was done in both primary and secondary rhinoplasty. The bony septum was used as extended spreader graft. Vomer or perpendicular plate of the ethmoid was harvested approximately one centimeter. Some studies ${ }^{2.3}$ have used septal bone, commonly vomer or perpendicular plate of the ethmoid, as batten graft in dorsal and caudal strut, aimed mostly at correction of caudal septal deviation (Figure 9). Another study ${ }^{4}$ used the bony septum as septal extension graft in cartilage-depleted patients. No study has yet described the use of bony septum as extended spreader graft.

Bone is more difficult to shape than cartilage and can be easily fractured while being shaped. We were able to obtain grafts with the aid of septal scissors. In settings where septal scissors is not available, Mayo scissors maybe used taking into account its bulkiness and difficulty in manipulation in a small area. Fixation of bone grafts to the L-strut also poses a challenge. Hence, burring of holes was done on either end of the graft to allow a precise and secure fixation of the graft. These holes also allow rapid ingrowth of vascularized tissue. The perpendicular plate of the ethmoid is thin, flattened and straighter while the vomer is more irregular. In this study, a septal extension graft using the central harvested septal cartilage is fixed to one side while an extended spreader graft on the contralateral side using bony septum. (Figure 4) A SEG is best secured with ESG because of its firm nature to hold the SEG in the desired vector. The bony septum widens the internal valve as well as supports the weak SEG although it is known to resorb in a year. Postoperatively, the patients were evaluated via anterior rhinoscopy. There was improvement in breathing noted subjectively by the patients.

As evident in the pre- and post-operative photographs taken for evaluation (Figure 5), control of the nasal length was achieved and tip deviation or rotation was corrected. Avoidance of post-operative nasal shortness is another long-term advantage.

The use of the bony septum (vomer and perpendicular plate of the ethmoid) as an extended spreader graft during primary or secondary rhinoplasty is a potentially effective method of supporting and securing the septal extension graft for preventing tip deviation or rotation and short-nose deformity, and for improving internal valve dysfunction. In our small series, it stabilized the septal extension graft and improved breathing, serving its purpose aesthetically and functionally. Further trials with a larger population and longer follow-up are needed to establish its reliability and long-term effectivity. The use of a zerodegree nasal endoscope is likewise recommended to facilitate assessment of internal valve collapse and post-operative improvement.

REFERENCES

1. Kim JH, Park SW, Song JW, Oh WS, Lee JH. Effective septal extension graft for asian rhinoplasty. Arch Plast Surg. 2014 Jan; 41(1):3-11. DOI: 10.5999/aps.2014.41.1.3; PMID: 24511488 PMCID: PMC3915153.

2. Dini GM, lurk LK, Ferreira MC, Ferreira LM. Grafts for straightening deviated noses. Plast Reconstr Surg. 2011 Nov;128(5): 529e-537e. DOI: 10.1097/PRS.0b013e31822b6989; PMID: 22030515.

3. Jang YJ, Kim JM, Yeo NK, Yoo JH. Use of nasal septal bone to straighten deviated septal cartilage in correction of deviated nose. Ann Otology Rhinol Laryngol. 2009 Jul; 118(7):488-494. DOI: 10.1177/000348940911800706; PMID: 19708487.

4. Kim GR, Park K, MD, Kim T. Use of nasal septal bone for septal extension graft after jaw surgery. Plast Reconstr Surg Glob Open. 2013 Dec; 1(8): e76. DOI: 10.1097/GOX.0000000000000016; PMID: 25289271; PMCID: PMC4186304.

5. Baladiang DE, Olveda MB, Yap EC. The "sail" excision technique: a modified alar lift procedure for Southeast Asian noses. Philipp J Otolaryngol Head Neck Surg. 2010 Jan-Jun; 25(1): 31-37.

6. Yap EC. Aesthetic Rhinoplasty for Southeast Asians. In: Jin HR (editor). Aesthetic Plastic Surgery of the East Asian Face. New York: Thieme; 2016. p. 109-110.

7. Lee KJ. Essential Otolaryngology: Head and Neck Surgery, $10^{\text {th }}$ ed. New York: Mc Graw Hill; 2008. p745.

8. Courtiss EH, Goldwyn RM. The effects of nasal surgery on airflow. Plast Reconst Surg. $1983 \mathrm{Jul}$; 72(1):9-21. PMID: 6867182.

9. Kim $\mathrm{YH}$, Jang TY. Porous high-density polyethylene in functional rhinoplasty: excellent longterm aesthetic results and safety. Plast Surg (Oakv). 2014 Spring; 22(1):14-7. PMID: 25152641 PMCID: PMC4128427.

10. Sheen JH. Spreader graft: a method of reconstructing the roof of the middle nasal vault following rhinoplasty. Plast Reconst Surg. 1984 Feb; 73(2):230-9. PMID: 6695022.

11. Hilinski JM, Byrne P. Spreader Grafts Rhinoplasty. Medscape. Updated: 2016 Feb 05.

12. Palacín JM, Bravo FG, Zeky R, Schwarze H. Controlling nasal length with extended spreader grafts: a reliable technique in primary rhinoplasty. Aesthetic Plast Surg. 2007 Nov-Dec; 31(6):645-50. DOI: 10.1007/s00266-007-0065-8; PMID: 17876658.

13. Schlosser RJ, Park SP. Surgery for the dysfunctional nasal valve. Cadaveric analysis and clinical outcomes. Arch Facial Plast Surg. 1999 Apr-Jun; 1(2):105-110. PMID: 10937087.

14. Suh MW, Jin HR, Kim JH. Computed tomography versus nasal endoscopy for the measurement of the internal nasal valve angle in Asians. Acta Otolaryngol. 2008 Jun; 128(6):675-9. DOI: 10.1080/00016480701663391; PMID: 18568504.

15. Heinberg CE, Kern EB. The Cottle sign: an aid in the physical diagnosis of nasal airflow disturbances. Rhinology. 1973; 11:89-94. 